\title{
EVOLUCIÓN RECIENTE DE LA POBLACIÓN DE LA MANCHA OCCIDENTAL
}

\author{
POR \\ RAÚL ROMERO CALCERRADA \\ $\mathrm{Y}$ \\ JAVIER MARTÍNEZ VEGA
}

\section{Introducción}

La población, sus características, su evolución y sus tomas de decisiones repercuten en la fisonomía de un lugar y, por tanto, en su paisaje. Partiendo de esta premisa, se pretende examinar algunas de las características de la población de la Mancha Occidental, principalmente en los dos últimos decenios. De esta forma, se espera que esta información ayude a comprender un poco mejor la evolución y procesos que han ocasionado el grave deterioro del acuífero 23 y de los espacios naturales asociados a éste. Se debería así complementar otras investigaciones sectoriales centradas en aspectos biofísicos de este espacio geográfico, tal y como demandan crecientemente las oficinas internacionales que coordinan investigaciones sobre los procesos de cambio global. Numerosos científicos consideran cada vez más relevante la información demográfica y económica para comprender, bajo una perspectiva integrada, el

Raúl Romero Calcerrada. Instituto de Economía y Geografía. CSIC. Madrid.

Javier Martínez Vega. Instituto de Economía y Geografía. CSIC. Madrid, y Universidad de Alcalá.

Estudios Geográficos

Tomo LVIV, n. ${ }^{\circ} 230$, enero-marzo, 1998 
problema suscitado por la sobreexplotación de los recursos hídricos y buscar una alternativa al desarrollo de la zona.

Somos conscientes de la arbitrariedad de las delimitaciones de acuíferos subterráneos ya que, en sí mismos, éstos no tienen por qué configurar una región homogénea. Este es el caso del Acuífero 23, objeto de estudio en este trabajo. Sus límites han sido establecidos, recientemente, por la Confederación Hidrográfica del Guadiana -Boletín Oficial de la Provincia de Cuenca $n .{ }^{\circ} 6,16$ de enero de 1995-. Sin embargo, la región manchega es mucho más amplia y engloba áreas que están sobre otros acuíferos adyacentes. Consideramos que los municipios asentados sobre el acuífero 23 definen bien lo que llamaremos a partir de ahora Mancha Occidental. Esta región (Fig. 1) está constituida por 40 municipios, repartidos en las provincias de Ciudad Real -26 municipios-, Cuenca - 11 municipios- y Albacete - 3 municipios-, abarcando unos $5.500 \mathrm{~km}^{2}$, delimitados por la poligonal del Acuífero 23 o unidad hidrogeológica 04-04. En el trabajo se han considerado municipios completos ya que éstos son la unidad de información estadística. Por esta razón, el área de estudio incluye, además de una porción de la llanura manchega - unidad fisiográfica dominante-, otros paisajes periféricos.

La topografía de la que se ha denominado Mancha Occidental se encuentra comprendida entre 500 y 1.200 metros de altitud, aproximadamente. La altitud media se sitúa entorno a los $600 \mathrm{~m}$. Dominan las llanuras y las pendientes suaves, interrumpidas en los bordes por las fuertes inclinaciones del piedemonte de las sierras periféricas donde se encuentran las mayores alturas.

La Mancha Occidental está constituida principalmente por materiales terciarios - principalmente calizas- cubiertos en algunas áreas por sedimentos pliocuaternarios y cuaternarios (aluvial, coluvial...). La caliza es determinante sobre la hidrología superficial y subterránea, dando origen al acuífero 23 y reduciendo los caudales que surcan su superficie. En las estribaciones de los Montes de Toledo encontramos materiales paleozoicos.

El clima es mediterráneo templado, con verano cálido y seco. En conjunto las precipitaciones medias son moderadas y varían entre los 400 $\mathrm{mm}$ y $600 \mathrm{~mm}$. La temperatura media anual presenta valores entre $12^{\circ}$ y $16^{\circ}$. El clima presenta, además, la degradación continental propia del interior peninsular que se traduce en la posibilidad de heladas durante un período de, al menos, 2 meses. 


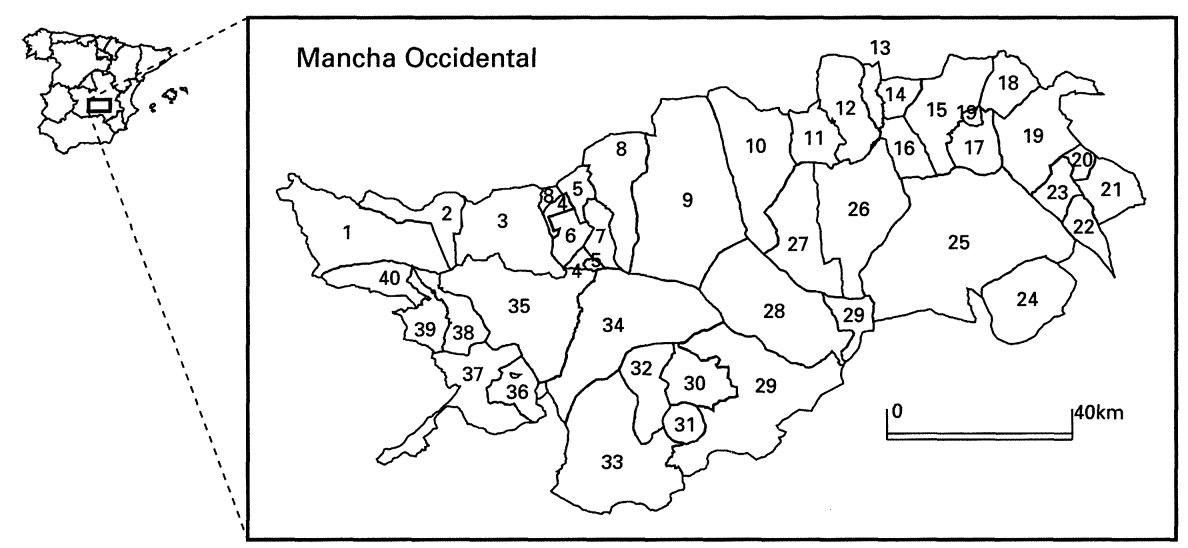

Los números en el mapa corresponden a los siguientes municipios:
$\begin{array}{lll}\text { 1. Malagón (CR) } & \text { 15. Las Pedroñeras (CU) } & \text { 29. Alhambra (CR) } \\ \text { 2. Fuente el Fresno (CR) } & \text { 16. Las Mesas (CU) } & \text { 30. La Solana (CR) } \\ \text { 3. Villarrubia de los Ojos (CR) } & \text { 17. El Provencio (CU) } & \text { 31. San Carlos del Valle (CR) } \\ \text { 4. Las Labores (CR) } & \text { 18. Alberca de Záncara (CU) } & \text { 32. Membrilla (CR) } \\ \text { 5. Puerto Lápice (CR) } & \text { 19. San Clemente (CU) } & \text { 33. Valdepeñas (CR) } \\ \text { 6. Arenas de San Juan (CR) } & \text { 20. Casas de Fernando Alonso (CU) } & \text { 34. Manzanares (CR) } \\ \text { 7. Villarta de San Juan (CR) } & \text { 21. Casas de Haro (CU) } & \text { 35. Daimiel (CR) } \\ \text { 8. Herencia (CR) } & \text { 22. Minaya (ALB) } & \text { 36. Bolaños de Calatrava (CR) } \\ \text { 9. Alcázar de San Juan (CR) } & \text { 23. Casas de los Pinos (CU) } & \text { 37. Almagro (CR) } \\ \text { 10. Campo de Criptana (CR) } & \text { 24. Munera (ALB) } & \text { 38. Torralba de Calatrava (CR) } \\ \text { 11. Pedro Muñoz (CR) } & \text { 25. Villarrobledo (ALB) } & \text { 39. Carrión de Calatrava (CR) } \\ \text { 12. Mota del Cuervo (CU) } & \text { 26. Socuéllamos (CR) } & \text { 40. Fernancaballero (CR) } \\ \text { 13. Santa María de los Llanos (CU) } & \text { 27. Tomelloso (CR) } & \\ \text { 14. El Pedernoso (CU) } & \text { 28. Argamasilla de Alba (CR) } & \\ & & \end{array}$

Los suelos más comunes que se encuentran en la región son los Regosoles, Cambisoles, y sobre todo, Calcisoles, dominantes en la mayor parte de la Mancha Occidental.

La vegetación climácica son las quercíneas; sin embargo, las asociaciones clímax han sido sustituidas, de manera generalizada, por sus etapas seriales de matorral arbolado, matorrales y pastizales. El intenso aprovechamiento agrario de estas tierras ha ocasionado la desaparición de la vegetación natural, viéndose reducida a las áreas de montaña y zonas marginales. En las áreas agrícolas encontramos algunas encinas formando dehesas.

En la región se desarrollaba tradicionalmente la trilogía mediterránea - vid, cereal y olivos-, complementada con cultivos de regadío. En la década de los 80 , aquella trilogía se vio desequilibrada por la expansión de nuevos cultivos en regadío. Éstos llegaron a alcanzar las 120.000 ha.

$$
-77-
$$


El sector ganadero se encuentra constituido por pequeñas y medianas explotaciones de bovino, ovino, caprino, porcino y granjas avícolas.

Las principales industrias manchegas - de vino, queso y aceite- se encuentran vinculadas al agro. El sector de la construcción se encuentra bastante desarrollado, desplazando un importante número de obreros a Madrid, fundamentalmente. Ello permite el mantenimiento de las actividades agrarias a tiempo parcial y un importante complemento a sus economías.

Indudablemente los importantes condicionantes del medio físico, repercuten en la evolución y desarrollo de la agricultura de la región. Inevitablemente esta imbricación hombre-medio ocasiona que la propia sociedad padezca los efectos negativos causados por el modelo de agricultura insostenible, implantado en las últimas décadas.

\section{La evolución poblacional}

Los cambios en la distribución espacial de la población así como su evolución desde mediados de siglo resultan interesantes. Aunque para obtener una visión más global estudiaremos la evolución de la población a lo largo del siglo, nos centraremos en un período temporal más cercano a nosotros, desde mediados de siglo. En primer lugar, porque es el momento de inflexión de la curva y comienzan fenómenos como la emigración y ralentización del crecimiento. En segundo lugar, y más importante, porque coincide con un período de grandes transformaciones agrarias en la que se ha pasado de una agricultura tradicional a una intensiva.

La evolución de la población de la Mancha Occidental posee similitudes con la española. En general existe una tendencia ascendente hasta los años 50, a partir de los cuales, se advierte un descenso paulatino. No obstante, a partir de 1981 también es cierto que se atisba una ligera o tímida recuperación en muchos de los municipios manchegos.

Esta región ha estado sometida a un largo proceso migratorio o éxodo rural - debido a una estructura económica débil y a su cercanía a lugares dinámicos- cuya máxima intensidad se alcanzó en los últimos cincuenta años. La emigración tuvo cotas muy elevadas en los años 60 y 70 , comenzando su reducción a partir de los setenta, debido a las difi- 
cultades laborales que la crisis económica ocasionó en los centros de atracción, Madrid, Cataluña y País Vasco.

Si examinamos detalladamente los municipios manchegos podemos establecer tres grupos: municipios cuyas poblaciones sufren un incremento de sus efectivos hasta los años 50 para perderlos a partir de ese momento, como consecuencia de la emigración a Madrid, Barcelona, etc. Los municipios que forman parte de este grupo son un total de 18 de los 40 que se encuentran situados sobre el acuífero 23. Los municipios más pequeños son los más numerosos en este grupo y cuanto menor población poseen más exageradas presentan las tendencias. Como sabemos, son las escasas perspectivas de futuro las que incitaban a la movilidad de la población. El único municipio importante que encontramos en este grupo es Socuéllamos, aunque tiene una fase regresiva muy suave.

El segundo grupo está formando por los municipios que han mantenido una estabilidad demográfica en todo el período estudiado. Se encuentra situado en la zona Oeste-Suroeste del Acuífero 23, próximo a núcleos urbanos dinámicos. Dentro de este grupo distinguimos dos tendencias o subgrupos: el primero está caracterizado por el equilibrio, como en el caso de Almagro y Valdepeñas. Ambos municipios presentan estabilidad poblacional salvo un pequeño aumento a mediados de siglo. Esto puede ser debido a su importancia como capitales comarcales, sobre todo de Valdepeñas, y a su proximidad a dos importantes ciudades como son Ciudad Real y Puertollano. El segundo subgrupo está constituido por Carrión y Torralba de Calatrava. Ambos presentan un equilibrio hasta los años 50 para sufrir un rápido descenso, que dura hasta 1970 y 1981 respectivamente, equilibrándose nuevamente. Estos municipios presentan características muy similares, con poblaciones entre 4.000 y 5.000 habitantes hasta los años 50 y entre 2.500 y 3.000 en los años 80, cercanía mutua y proximidad a Ciudad Real.

El tercer grupo está integrado por municipios con tendencia a aumentar el número de habitantes. Al igual que el grupo anterior puede desdoblarse en dos subgrupos: uno que ha alcanzando cierto equilibrio y otro que ha mantenido esa tendencia. Los municipios que aumentaron su población hasta los años 50 y 60 para mantener luego un equilibrio son Alcázar de San Juan, Argamasilla de Alba, Campo de Criptana, Las Mesas, Manzanares, Membrilla, Pedro Muñoz, San Clemente, La Solana, Tomelloso, Villarrobledo, Villarta de San Juan y Vi- 
llarrubia de los Ojos. Como vemos, se incluyen en él la mayor parte de los núcleos importantes de esta región y que acusan la crisis económica. El otro subgrupo es aquel que mantiene la tendencia ascendente hasta la actualidad. Se incluyen Bolaños de Calatrava, Mota del Cuervo y Las Pedroñeras, municipios todos ellos que hoy en día tienen un importante dinamismo demográfico.

Una vez examinada la población a lo largo del siglo xx, examinaremos la variación de los porcentajes de población de cada municipio entre 1950 y 1991. En la Figura 2 se puede observar una dualidad: en primer lugar, sobresalen los municipios que han experimentado grandes descensos, localizados en la zona occidental del acuífero 23, próximos a la capital y a otros núcleos importantes de población. Éstos actúan como polos de atracción, absorbiendo la población de los municipios más cercanos e incluso como trampolín hacia las grandes ciudades. Los núcleos de población situados próximos a la capital provincial han perdido un porcentaje superior al 0,4\%. Hacia el Este manchego, Minaya, El Provencio, Herencia y Alhambra participan del mismo proceso.

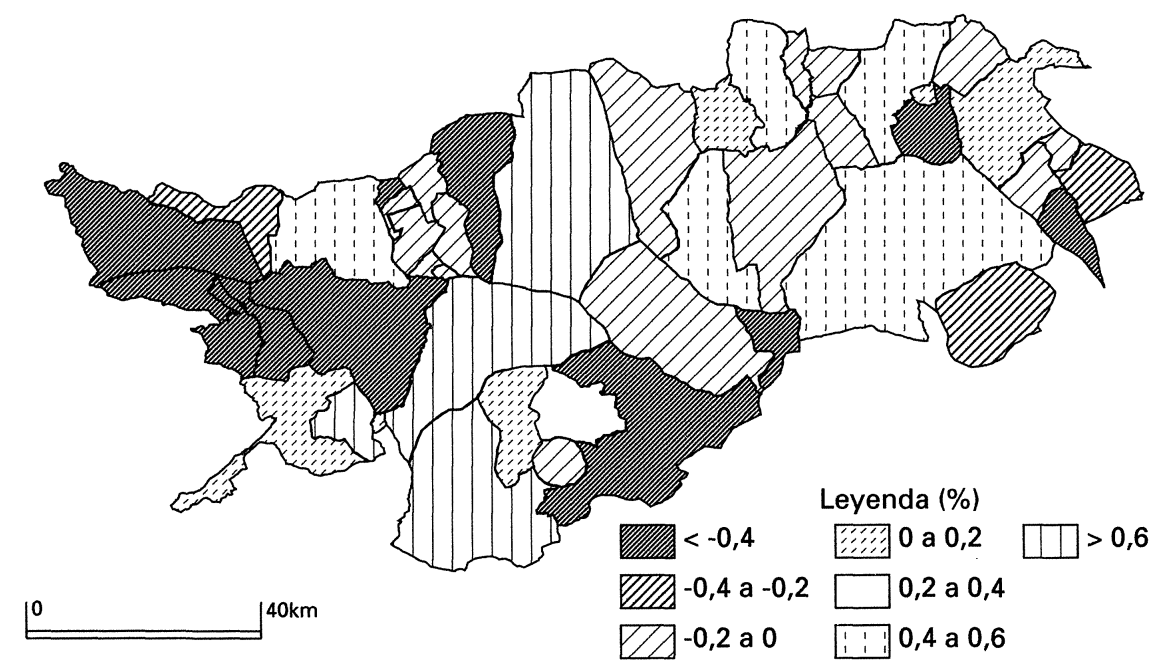

Fuente: Censos de población. Elaboración propia.

FIgURA 2.-Evolución de la población en el periodo intercensal 1950-1991. 
En el polo opuesto, los pueblos que juegan un papel importante, como centros administrativos, de servicios o industriales son los únicos que han mantenido y aumentado la población, seguramente a costa de los municipios vecinos. Destaca el eje de Alcázar de San Juan, Bolaños de Calatrava y Valdepeñas por su considerable incremento en el conjunto manchego. No obstante, Villarrobledo, Mota del Cuervo, Las Pedroñeras y Tomelloso, también han incrementado sus efectivos, aunque más ligeramente, sin superar el índice de $0,6 \%$.

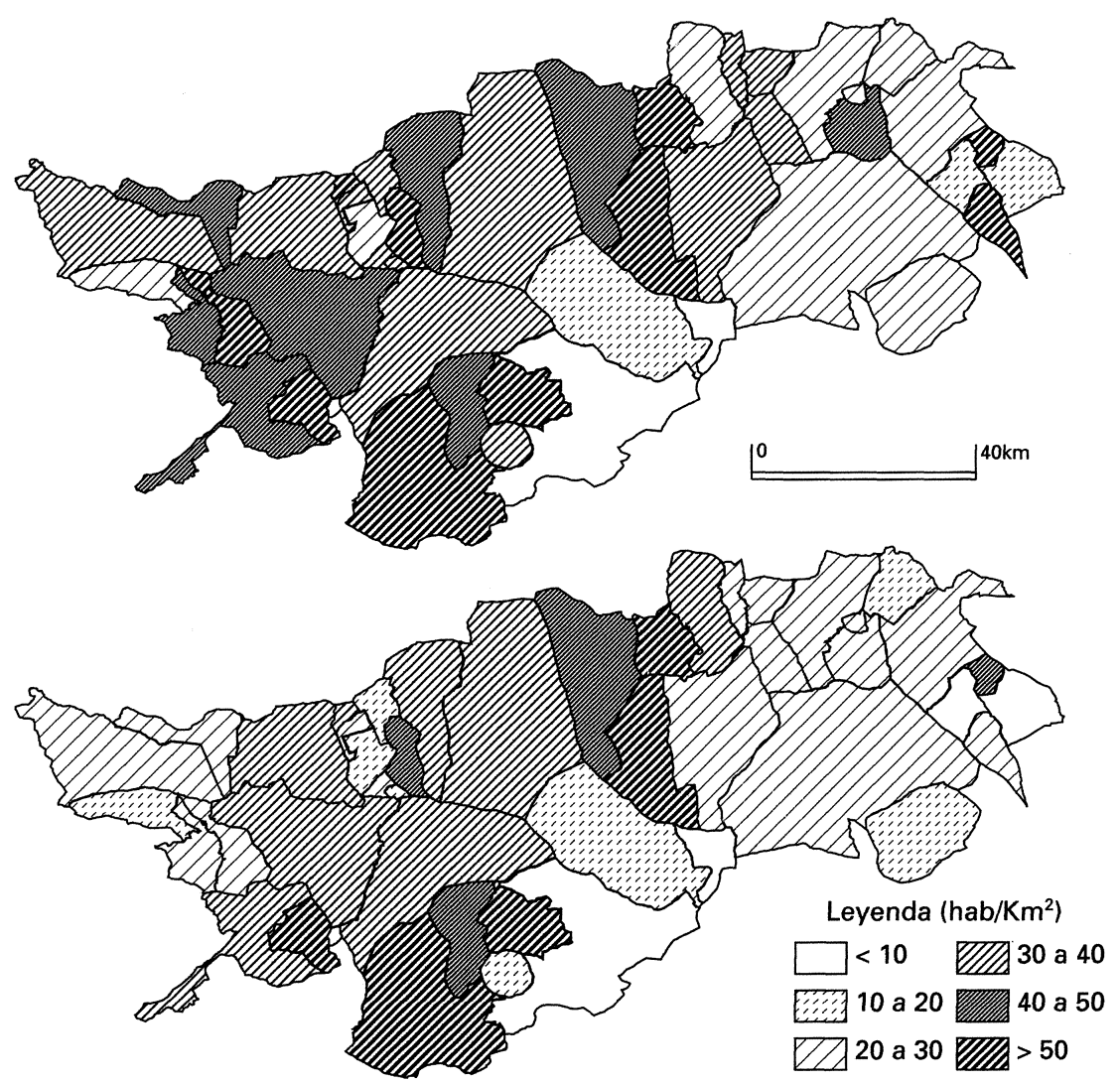

Fuente: Censos de población. Elaboración propia.

FIGURA 3.-Densidad de población en 1950 (arriba) y 1991 (abajo). 
La Figura 3 resulta muy significativa para ilustar el proceso despoblador sufrido en todo el área. En 1950 apreciamos cómo la mayor parte de los municipios poseen densidades comprendidas entre 30 y 50 hab. $/ \mathrm{km}^{2}$, siendo los municipios de Cuenca, Este y Sur-Este los de menores densidades. En 1991 se constata un fuerte retroceso de las densidades, más acentuado en los bordes Oriental y Occidental. Los municipios albacetenses y conquenses registran un fuerte despoblamiento, generalizándose por toda la zona valores promedios entre 20 y 30 hab. $/ \mathrm{km}^{2}$, cifra inferior a la de 1950. La zona Occidental del acuífero es quizás la más expresiva de esta transformación, pasando de valores de 40 a 50 hab. $/ \mathrm{km}^{2}$ a mediados de siglo a valores inferiores a los 30 hab. $/ \mathrm{km}^{2}$ en 1991. La zona central ha permanecido estable en todo este tiempo. Los núcleos de mayor densidad son Campo de Criptana, Pedro Muñoz, Tomelloso, La Solana, Membrilla y Valdepeñas. Esta dualidad se corresponde, en gran medida, con la temprana crisis del regadío en la zona Occidental, donde antes comenzó a notarse la falta de agua para regar. Por el contrario, la concentración de grandes propietarios en los municipios centrales ocasionó la perduración de una próspera agricultura intensiva de regadío gracias a la posibilidad de profundizar más y mantener los caudales necesarios. Es posible que este hecho haya contribuido a fijar parte de la población. Junto a este factor existen otros convergentes. Es en estos municipios centrales donde se concentra la industria vinculada a los sectores agroalimentario y metalúrgico.

En el Cuadro I observamos que mientras que en España se ha producido un aumento considerable de la densidad, en la zona se manifiesta un descenso de $8 \mathrm{hab} . / \mathrm{km}^{2}$.

CUADRO I

DENSIDAD DE POBLACIÓN ENTRE 1950-1991

\begin{tabular}{c|c|c|c}
\hline Hab./km ${ }^{\mathbf{2}}$ & Acuífero 23 & Castilla-La Mancha & España \\
\hline 1950 & 43 & 26 & 55 \\
1991 & 35,78 & 21 & 78 \\
\hline
\end{tabular}

Fuente: Censos de población. Elaboración propia.

Así pues, la Mancha Occidental no presenta ninguna excepción dentro del modelo de redistribución centro-periferia. Esta región -inte- 
rior - pierde población en favor de áreas litorales más dinámicas, lo que ha llevado al descenso del ritmo de crecimiento de la población.

No obstante, conviene decir que si se comparan los valores estadísticos de la población estudiada con los de Castilla-La Mancha es evidente que nos encontraremos en una de las comarcas más dinámicas de la región. Este territorio, que superficialmente supone cerca del 7\% del total regional, concentraba en 1991 casi el 25\% de la población total de Castilla-La Mancha. Este hecho da idea del peso específico de la población de la Mancha Occidental en la toma de decisiones del gobierno regional.

\section{El movimiento natural de la población}

En España, la evolución del movimiento natural de la población sigue rumbos parecidos a los experimentados por otras sociedades avanzadas. Actualmente, presenta unos índices de nupcialidad, fecundidad, mortalidad y crecimiento natural bajos. La transición demográfica, realizada de forma vertiginosa, parece haber entrado definitivamente en su recta final, sobre todo en lo que a natalidad se refiere.

Hasta 1970, la situación de Castilla-La Mancha se enmarcaba dentro del proceso general de cambio que se estaba desarrollando en España. En la década de los 70, se produjeron grandes alteraciones, ligero aumento de la mortalidad, descenso paulatino de la natalidad... ocasionando el decrecimiento natural de la población, que Estébanez (1979) atinadamente puso de manifiesto. Después de 1975 es cuando la situación más ha cambiado, si bien la evolución parece coincidir, en términos generales, con la experimentada en España. El problema se manifiesta, en sus verdaderas dimensiones, cuando se analizan los factores naturales y el crecimiento vegetativo a escala municipal.

Del examen del movimiento natural de la población desde 1975 a 1991, apreciamos, en la mayoría de los municipios, una tendencia hacia el decrecimiento, salvo en algunos casos puntuales donde existe un equilibrio muy precario. Está claro que una serie más amplia podría haber ayudado a extraer conclusiones más firmes, sin embargo ésta confirma la hipótesis que ya imaginábamos.

En todos los municipios de la Mancha Occidental se comprueba que el número de nacimientos se ha ido reduciendo, en algunos de for- 
ma constante como Alcázar de San Juan, Campo de Criptana y Villarrobledo, entre otros. Mientras el número de matrimonios se ha mantenido en estos 16 años, lo que evidencia un ajuste a las tendencias natalistas modernas y un control de la natalidad, el número de muertes ha ido aumentando ligeramente. Todo ello corrobora que el incremento poblacional de algunos de estos municipios se debe a la inmigración desde núcleos cercanos.

Junto a estos municipios, en los que el crecimiento vegetativo es negativo, encontramos otros -Almagro, Tomelloso o Valdepeñasdonde se aprecia cierta estabilidad de todas las variables. Existe un paralelismo entre el número de nacimientos, defunciones y matrimonios $\mathrm{y}$, por tanto, un crecimiento vegetativo estable. En ellos, tanto el aumento de población como su disminución se explica por factores no naturales.

En general, podemos concluir afirmando que la situación demográfica en Castilla-La Mancha es crítica y que el proceso regresivo en el crecimiento natural de la población se está afianzando día a día incrementándose el número de municipios con tasas negativas así como la magnitud de las mismas. En 1981, eran 5 municipios los que registraban crecimientos vegetativos negativos mientras que, en 1991, aumentaban a 10 núcleos.

\section{Estructura demográfica por edades}

El estudio de las estructuras demográficas constituye un tema de interés porque espacialmente su reparto es desigual y ello provoca importantes consecuencias biológicas, sociales, económicas y culturales.

De acuerdo a los datos del Censo de 1981, España tenía una tasa de juventud del 243,59 , un índice de vejez de 41,05 y una tasa de dependencia del 63,02. Según los datos de 1991, España ha reducido su tasa de juventud hasta 152,83 y su tasa de dependencia a 53,67 mientras que el índice de envejecimiento creció hasta 65,43.

En la Figura 4 podemos apreciar cómo en 1981 el numero de jóvenes es elevado en la mayor parte de los municipios de la Mancha Occidental - la media de la comarca es de 211 jóvenes por cada 100 viejos-, alcanzándose en 19 de ellos tasas superiores a 220. Si lo comparamos con los valores medios nacionales, se observa que tan sólo 7 municipios están próximos a ellos lo que trasluce la menor dinámica natural, propia de 


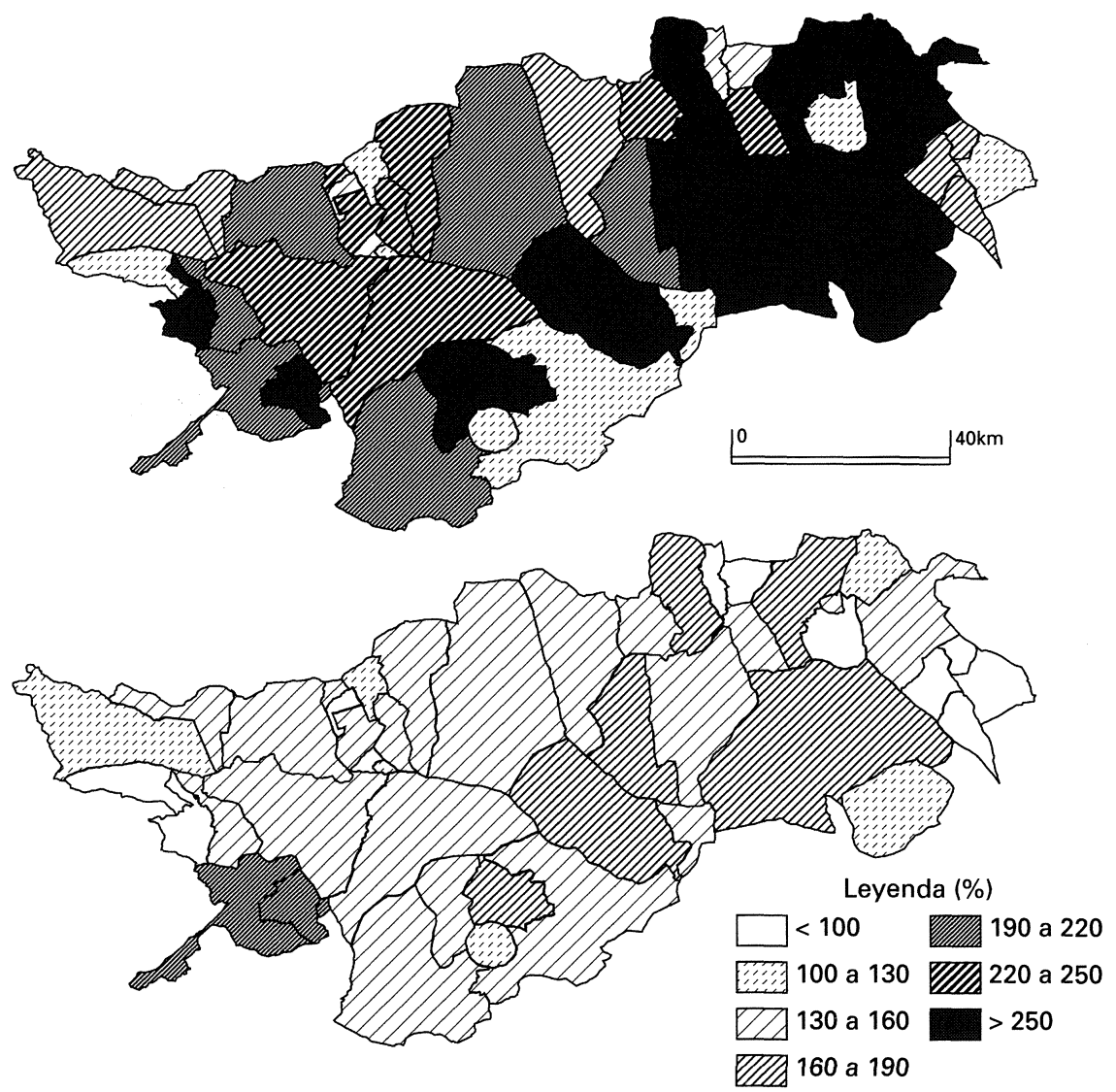

Fuente: Censos de población. Elaboración propia.

FIGURA 4.-Indice de juventud en 1981 (arriba) y 1991 (abajo).

las poblaciones del mundo rural afectado por las migraciones. La situación en 1991 varía, siendo ahora 18 los municipios con valores próximos a la media nacional. Sin embargo, existen casos extremos en los que se cuenta con tasas de juventud elevadas - de 190 a 220 registran Bolaños de Calatrava y Almagro- o en los que los efectivos de ancianos superan a los jóvenes - Casas de Fernando Alonso, Casas de Haro, Torralba de Calatrava y Fernáncaballero-. Los municipios del área estudiada registran un valor medio, en 1991, de 133 jóvenes por cada 100

$$
-85-
$$


viejos. En resumen, toda la región ha sufrido, en 10 años, un importante descenso - 78 jóvenes por cada 100 viejos- de sus bases demográficas.

El estudio de la estructura por grandes grupos de edad viene a ratificar que el grupo de jóvenes (0-14 años) ha reducido sus porcentajes en todos los casos, a excepción de Almagro y Bolaños de Calatrava que los han mantenido. Este hecho, casi general en la zona, coincide con la evolución seguida a nivel nacional. Resulta preocupante observar el fuerte descenso del número de jóvenes en tan poco tiempo, hasta tal punto que el reemplazo generacional y la fuerza de trabajo podrían verse seriamente comprometidos.

En 1981, la Mancha Occidental poseía un índice de envejecimiento de 52 , que indicaba ya una población envejecida. Durante el último decenio la zona ha sufrido un incremento del número de ancianos. En 1991, el índice de vejez regional es realmente alto, con 82 viejos por cada cien jóvenes. El número de municipios que poseen valores similares a la media nacional es de 16; el resto posee valores muy superiores, duplicándolos incluso - 130 viejos por cada 100 jóvenes-.Éstos son los más cercanos a las capitales provinciales, como Fernáncaballero, Casas de Haro, Minaya, etc. En resumen, es evidente que la región ha sufrido un fuerte envejecimiento, sin duda, debido a dos fenómenos: la emigración y los desequilibrios demográficos.

Como síntesis, la Figura 5 representa la Tasa de Dependencia. En ella se observa cómo las tasas de dependencia se han visto reducidas considerablemente de una fecha a otra, con valores medios en la Mancha Occidental de un -4\%. No obstante, España posee un ritmo de decrecimiento mucho mayor, cercano al -10\% en el mismo período. En 1981 existían 17 municipios que se aproximaban a los valores nacionales mientras que, diez años después, tan sólo Casas de Fernando Alonso y Casas de los Pinos poseen valores similares a los nacionales, teniendo el resto tasas superiores a la media nacional de 1991 (53,67\%). Una de las causas de este hecho es que el descenso de jóvenes es más acusado que el incremento de viejos.

En general, el descenso del número de personas dependientes provoca un situación buena a corto plazo, ya que el colectivo poblacional de adultos es elevado, y por tanto, también lo es la fuerza laboral. Asimismo, se reduce la carga impositiva para los activos. No obstante, a medio plazo, es evidente que el envejecimiento de la población tornará esta si- 
tuación coyuntural hacia sendas preocupantes Un nuevo incremento de las tasas de dependencia y no disponer de la mano de obra suficiente para la explotación de los recursos productivos son algunas consecuencias directas previsibles.

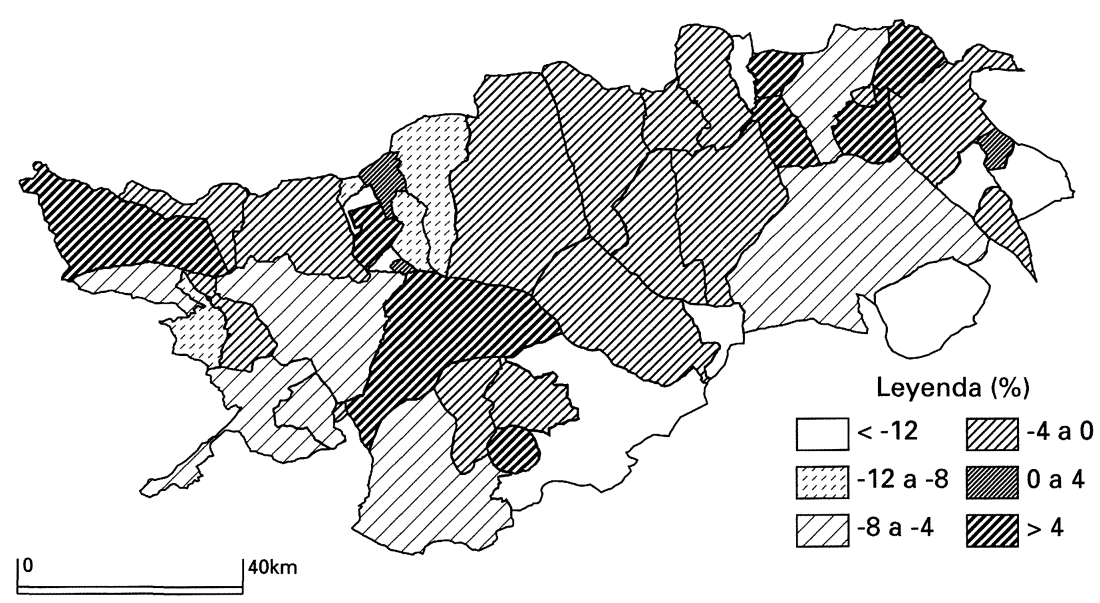

Fuente: Censos de población. Elaboración propia.

Figura 5.-Evolución de la tasa de dependencia en el período intercensal 1981-1991

\section{Estructura de la población}

La consideración de la variable actividad en el análisis de una población dada no forma parte, en sentido estricto, del estudio de las estructuras demográficas, puesto que, para un conjunto humano cualquiera, este aspecto sólo indirectamente es relacionable con la dinámica del colectivo de que se trate. Sin embargo, el estudio de las características de la fuerza de trabajo es la principal motivación de la demografía, y por ello un aspecto siempre primordial del conocimiento de las estructuras poblacionales.

Como en apartados anteriores conviene interpretar las tasas de actividad comparándolas con la de otros ámbitos que sirvan de marco de referencia. A nivel nacional, la tasa de actividad, según el Censo de 
1981, era para los hombres del $73,12 \%$ y del $22,37 \%$ para las mujeres. En 1991 , mientras que se ha reducido a $68,32 \%$ la tasa masculina, se constata un incremento de la participación de las féminas en el mundo laboral $(33,26 \%)$.

En el examen de la evolución de las tasas de actividad por sexos entre 1981 y 1991 - se observa que la tendencia de las tasas de actividad masculina en la Mancha Occidental es similar a la del resto del país. Es decir, se aprecia una progresiva disminución de sus valores, lo que supone una pérdida de efectivos superior al 10,5\%. El comportamiento de las mujeres activas difiere notablemente. Su característica fundamental es la lenta elevación - un 8\% - de sus tasas específicas. La mujer se incorpora al trabajo, de una forma progresiva, pero a un ritmo inferior al nivel nacional. La razón, quizás, estribe en la propia estructura productiva del área estudiada. Es sabido que la mujer manchega participa activamente en las tareas agrícolas, especialmente como ayuda familiar en los momentos en que más falta hace: la recolección. Sin embargo, no siempre son contabilizados-declarados estos puestos de trabajo. Por otra parte, el tejido industrial, comercial y de servicios no crece en la región al ritmo nacional por lo que las expectativas de una creciente incorporación de la mujer al trabajo se ven frenadas.

La Tasa de Actividad masculina de 1991 en la mayor parte de los municipios es bastante alta, superior al 70\%. Cerca de una tercera parte de los municipios manchegos poseen valores próximos a la media nacional (68,32\%), siendo el caso de Daimiel representativo (69\%). La parte más Occidental presenta, en general, valores de ocupación inferiores a los de la parte más Oriental, quizás debido a la ocupación de mano de obra en la construcción.

En la evolución de las tasas de actividad de los hombres entre 1981 y 1991, se aprecia un importante descenso. El valor promedio de la Mancha Occidental se sitúa en un $-5,53 \%$ superior al $-4,8 \%$ de la media nacional, registrado en igual período. En general, existen grandes disparidades a nivel municipal. No obstante, se entrevén algunas propensiones. Parece que los municipios próximos a las capitales mantienen su actividad e, incluso, aquellos centros de servicios disminuyen poco la misma - por ejemplo, Daimiel -2,4\%-. Por el contrario, los municipios más industrializados -Manzanares, Alcázar de San Juan, etc. - han visto reducido el papel del hombre en el mercado 
de trabajo. Quedan otros municipios agrícolas que han reducido drásticamente sus cuotas de actividad masculina que son más difícilmente explicables.

A diferencia de los hombres las tasas de actividad femenina en 1991 son bajas, con valores que oscilan entre 10 y $24 \%$. Esta «feminización» de la actividad no tiene en todos los municipios la misma importancia (Puyol, 1989). Los valores más altos los encontramos en aquellos municipios fuertemente agrícolas — por la ayuda de la mujer en las labores del campo- o donde la industria y el sector servicios está más desarrollado. A pesar de todo, si comparamos los valores municipales con los nacionales podemos apreciar cómo frente al 33,3\% de tasa española de actividad femenina, tan sólo 5 municipios - Valdepeñas, Alcázar de San Juan, Daimiel, Malagón y Carrión de Calatrava - se acercan ligeramente a ese valor.

En la evolución de la tasa de actividad queda presente cómo en los últimos 10 años la mujer se ha introducido en el mercado de trabajo. Éste es uno de los aspectos más significativos. El trabajo de la mujer ha sufrido un aumento más homogéneo en toda el área de estudio y más próximo al 10,9\% de incremento a nivel nacional. Si bien se parte de diferencias significativas, la incorporación de la mujer al mercado laboral se va realizando de forma progresiva y al mismo ritmo nacional.

Conviene ahora examinar el reparto de la población activa por sectores económicos. Castilla-La Mancha ha sufrido profundamente una crisis de la sociedad agraria tradicional, plasmada, entre otras, en una reducción de su población ocupada en la agricultura. Ha conocido una reestructuración bastante notable en los sectores productivos a favor de la población, ocupada en el sector secundario (VV.AA. 1986b).

En la Figura 6 se observa un fuerte descenso de la población agraria manchega durante la última década. En 1981 se aprecian fuertes contrastes a nivel municipal, desde los núcleos fuertemente agrarios hasta los de avanzada implantación industrial —por ejemplo, Alcázar de San Juan-. Existe una elevada correspondencia entre los municipios que poseen mayor protagonismo económico con los que tienen menor población empleada en el sector primario. A pesar de ello, el valor medio de población dedicada a la agricultura en el conjunto de la Mancha Occidental es de 47,69\%. Comparando este valor con el 15,7\% nacional nos podemos hacer una idea del fuerte peso que todavía tenía el sector agrario en La Mancha. 


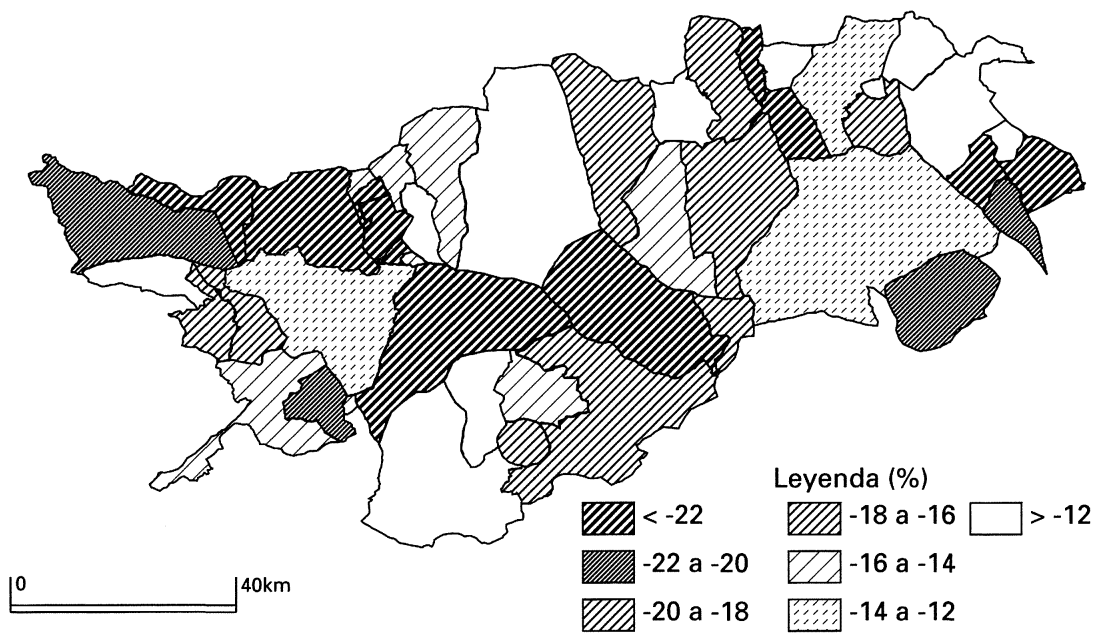

Fuente: Censos de población. Elaboración propia.

FIGURA 6.-Evolución de la población ocupada en la agricultura en el período intercensal 1981-1991.

En 1991, la situación ha variado considerablemente. Siguiendo las tendencias anteriormente descritas, existe un fuerte descenso de la población dedicada a la agricultura. Ahora la media de la Mancha Occidental es del $28,85 \%$. Los municipios que se encuentran en áreas marginales y de montaña son los que conservan un mayor porcentaje de población activa agraria, superior al $30 \%$. El resto presenta valores inferiores siempre a la media comarcal, manteniéndose la relación de poblaciones industriales y administrativas con aquellos núcleos de menor número de empleados en este sector. Teniendo en cuenta que en 1991 la población española ocupada en la agricultura rondaba el 10\%, ahora los valores de los municipios manchegos presentan una mayor proximidad, si bien, aún son elevados.

En la Figura 6 se aprecia la evolución experimentada por la población ocupada en la agricultura entre 1981 y 1991. Lo que llama más la atención es la magnitud del retroceso. Los descensos analizados en La Mancha oscilan entre $-10 \%$ y $-24 \%$, muy superiores a la media española $(-5,5 \%)$. Esto nos indica el enorme cambio sufrido en esta zona, superando en el más estable de los casos el doble de la reducción habida 
a nivel nacional. Son los municipios más industrializados y los más marginales, por situarse en áreas de montaña, los que menos población han perdido en este sector durante este período, seguramente porque la habían perdido anteriormente por reconversión o emigración. Por el contrario, los municipios de fuerte base agraria, situados próximos a Albacete - Minaya - y en el Centro - Argamasilla de Alba - y Oeste Fuente el Fresno- de La Mancha Occidental, son los que han sufrido un fuerte retroceso, registrando valores que llegan a $-24 \%$. A pesar de todo, el peso de la población activa en la agricultura sigue siendo bastante importante en toda la región, pudiendo considerarla eminentemente agraria.

La población ocupada en otros sectores de actividad ha aumentado sus efectivos. Es el fruto de la reestructuración que han sufrido los sectores productivos a favor de la población ocupada en la industria y, principalmente, en la construcción. Del examen de la evolución de la población ocupada en la industria se aprecia que, salvo Carrión de Calatrava, La Solana y Membrilla, el resto de municipios manchegos han ganado población activa en este sector. Se atisba una doble tendencia; por un lado, un escaso crecimiento en aquellos municipios donde el sector industrial estaba ya fuertemente implantado o donde el tejido industrial es prácticamente nulo - áreas de montaña-. Por otro lado, son las áreas que han desarrollado su industria recientemente donde se produce un mayor crecimiento del empleo en la industria. A nivel nacional, en este período se ha producido un retroceso del $2,3 \%$ de la población activa en el sector industrial. La tendencia en la zona ha sido inversa a la española, aumentando el empleo en este sector. Conviene tener en cuenta, que la industria de La Mancha es fundamentalmente agroalimentaria, mucho menos afectada por la crisis que la industria pesada.

Quizás, más ilustrativa sea aún la evolución de la población empleada en la construcción. Es la parte Occidental del área de estudio la que ha sufrido un importante crecimiento, superior al 12\%, de la población activa en este sector. Una idea de su magnitud nos lo proporciona el hecho de que, mientras España incrementó su población ocupada en la construcción un 2,5\% en el último decenio, Villarrubia y Daimiel lo hicieron en un 15 y $12,4 \%$, respectivamente. El trasvase de activos desde la agricultura hacia la construcción ha sido muy significativo en todos los casos, hasta el punto de que podemos hablar de una importante transformación del campo manchego hacia una agricultura a tiempo par- 
cial. En cualquier caso, nos interesa destacar que la construcción acapara valores de ocupación superiores a la industria, por lo que la desagrarización no se ha visto correspondida por una industrialización. Por tanto, parece que asistimos a un trasvase de fuerza de trabajo excedentaria en agricultura a un sector favorecido por la coyuntura reciente. En el sector de la construccion, los trabajadores se desplazan diariamente desde Villarrubia, Daimiel, Fuente el Fresno, Herencia, Alcázar de San Juan... a otras provincias próximas e incluso a Madrid.

El sector terciario ha aumentado entre 1981 y 1991 según las tendencias españolas. Tan sólo una tercera parte de los municipios - los cercanos a Ciudad Real y Albacete- han crecido por encima de 8,27\% crecimiento nacional- - El resto de la Mancha Occidental sufre un crecimiento moderado con un valor promedio de $5,85 \%$, a diferencia de lo sucedido a nivel nacional (Puyol, 1989) donde, beneficiándose del retroceso experimentado por los otros dos sectores, ha mantenido constante su crecimiento.

\section{Impactos sociales y económicos sobre la población}

La Mancha Occidental no presenta grandes diferencias respecto a otras áreas del interior peninsular. Pérdida de población, envejecimiento y falta de dinamismo son sus rasgos más sobresalientes. La evolución demográfica de la zona es prácticamente paralela a la del conjunto del país hasta 1960. A partir de esta fecha se aprecia un descenso del ritmo de crecimiento de la población, que coincide con un incremento sustancial de otras regiones. Por tanto, si consideramos a la población como recurso es evidente que esta región se empobrece lo que a medio plazo puede bloquear cualquier intento de desarrollo.

Las complejas relaciones población-recursos resultan preocupantes en una región agraria con fuertes problemas económicos. En la década de los 80 se expandieron de forma considerable los cultivos en regadío, provocando negativos impactos ambientales. Desecación de cauces y humedales de gran interés ecológico de turberas y contaminación de las aguas son algunas manifestaciones de la degradación. La sobreexplotación del acuífero subterráneo que sustentaba los recursos agrarios ha ocasionado un uso insostenible de los mismos. Conviene investigar más a fondo el perfil sociológico de la población para comprender los

$$
-92-
$$


mecanismos desencadenados en el proceso de sobreexplotación que podría abocar indefectiblemente a la desertización. La reducción de las rentas como consecuencia de la vuelta al secano y, en el mejor de los casos, el incremento de los costes de los regadíos que aún permanecen, están ocasionando delicados impactos socioeconómicos no deseados. El endurecimiento del Régimen de Explotación del Acuífero 23 ha provocado un sentimiento de malestar social en la población como consecuencia del endeudamiento soportado durante los últimos años.

No obstante, es evidente la necesidad imperante de proponer nuevos usos agrícolas en el terrazgo manchego si se desea conservar el medio natural, alcanzando un desarrollo sostenible. Los nuevos usos que se atisban son, necesariamente, los cultivos de secano y aquellos en regadío que necesitan exiguas dotaciones hídricas.

Este hecho ocasiona una merma de la capacidad de sostener una población creciente con el mismo nivel de renta alcanzado en los años ochenta. El estado del bienestar se halla en entredicho. Resultan inquietantes tanto el fuerte descenso del número de jóvenes en poco más de 10 años como el fuerte envejecimiento. Estos desequilibrios, junto a la pérdida de potencialidad biológica, comienzan a producir un proceso de despoblación que afecta a la región manchega.

Los municipios de La Mancha que se sitúan sobre el acuífero 23 están dejando de ser una zona de reserva demográfica, pasando de tener un fuerte dinamismo poblacional a ser un área deprimida. Aquellos núcleos de fuerte dinamismo van dejando paso a municipios con recursos demográficos agudamente desequilibrados. El proceso de despoblamiento que lentamente se siente sobre La Mancha Occidental podría conllevar, en el futuro, un alto coste social, estructural y económico como consecuencia de la indisponibilidad de mano de obra suficiente para la explotación de los recursos productivos de la zona o para la implantación de otros nuevos. 


\section{BIBLIOGRAFÍA}

Alonso Antolín, M. ${ }^{\text {a }}$ C. y Somolinos Pérez, Q. (1983): «Movimientos migratorios en Castilla-La Mancha», En Almud, n. ${ }^{\text {os }}$ 7-8, pp. 125-143.

ARAgón, R. (1986): Panorama de la emigración española en Europa. Ministerio de Trabajo y Seguridad Social. Madrid.

CABo Alonso, A. (1983): «Transformaciones en el mundo rural español durante el último cuarto de siglo». En Coloquio Hispano-Francés sobre espacios rurales, tomo I. Abril. Ministerio de Agricultura, Pesca y Alimentación. Madrid. pp. 31-44.

CAMPO, S. DEL, y NAVARRO, M. (1987): Nuevo análisis de la población española. Ariel. Barcelona.

ESTÉBANEZ ÁlVAREZ, J. (1979): «El dinamismo demográfico durante el siglo xx en cinco provincias españolas de fuerte emigración». En Acta Geológica Hispánica. Homenatge a Lluís Solé y Sabaris. Barcelona. pp. 571-575.

GaRCía FERNÁNDEZ, P. (1985): Población de los actuales términos municipales. 1900-1981. Población de hecho según los censos. Instituto Nacional de Estadística. Madrid. 234 pp.

I.N.E. (1951): Anuario estadístico de España. 1951. Instituto Nacional de Estadística. Madrid.

- (1994a): Censo de Población y Viviendas de 1991. Tomo I. Resultados Nacionales. Características Generales de la Población.Instituto Nacional de Estadística. Madrid.

- (1994b): Censo de Población de 1991. Tomo III. Resultados Municipales. Características Generales de la Población. Vol. II. Castilla-La Mancha, Ceuta-Melilla. Instituto Nacional de Estadística. Madrid.

- (1996): Anuario estadístico. 1995. España. Instituto Nacional de Estadística. Madrid.

Molina IBÁÑEz, M. et al. (1988): Los movimientos migratorios en Castilla-La Mancha. Junta de Comunidades de Castilla-La Mancha. Toledo.

NADAL, J. (1984): La población española. Siglos XVI al XX. Ariel. Barcelona.

PANADERo Moya, M. (1980): «Demogeografía de la España interior», en Anales del Centro de Albacete, n. ${ }^{\circ}$ 2. Universidad Nacional de Educación a Distancia. Albacete, pp. 187-266.

- (coord.) (1982): Castilla-La Mancha: Espacio y Sociedad. Actas de la I Reunión de Estudios Regionales de Castilla-La Mancha, 3 vols. Junta de Comunidades de Castilla-La Mancha. Albacete.

PILLET CAPDEPÓN, F. (coord.) (1985): El espacio geográfico de la provincia de Ciudad Real. Diputación provincial de Ciudad Real, Ciudad Real.

- (1983): «Fluctuaciones en el crecimiento demográfico de la provincia de Ciudad Real y sus causas, 1867-1981». En Almud, n. os 7-8. Ciudad Real. pp. 193-205.

- (coord.) (1991): La provincia de Ciudad Real (I): GEOGRAFIA. Diputación provincial de Ciudad Real. Albacete.

PuYol Antolín, R. (1989): La población. Editorial Síntesis. Madrid.

PuYol, R.; EstébANEZ, J. y MÉNDEZ, R. (1988): Geografía Humana. Cátedra Geografía. Madrid.

RODRÍGUEZ DE LA TORRE, F. (1983): «Datos estadísticos sobre la población de la Comunidad de Castilla-La Mancha». En Almud, n. os 7-8. pp. 97-144.

RodrígueZ OsunA, J. (1985): Población y territorio en España. Siglos XIX y Xx. Espasa Calpe Madrid.

Romero CALCERRADA, R. (1996): La incidencia de los cambios de usos del suelo sobre un espacio natural: Las Tablas de Daimiel. Memoria de Licenciatura Inédita. Universidad de Alcalá de Henares, Madrid.

SÁEnz LoRITE, M. (1988): Geografía Agraria. Introducción a lso Paisajes Rurales. Editorial Síntesis. Madrid, p. 63.

VV.AA. (1986a): «Castilla-La Mancha». En El Campo. Boletín de Información Agraria. Bilbao, pp. 33-43.

- (1986b): Programa de Desarrollo Regional de Castilla-La Mancha 1986-1988. Consejería de Economía y Hacienda. Junta de Comunidades de Castilla-La Mancha. Toledo. 
RESUMEN: Evolución reciente de la población de la Mancha Occidental. La Mancha Occidental ha mantenido tendencias similares a la nacional hasta 1960. A partir de este momento se han iniciado tendencias divergentes. La región manifesta los primeros síntomas del proceso despoblador sufrido en otras regiones españolas. En este artículo examinamos algunas de las características principales de la población y su evolución reciente.

Palabras Clave: Mancha Occidental. Evolución de la población. Estructuras demográficas. Despoblación.

ABSTRACT: Recent evolution of the Mancha Occiental's population. The Mancha Occidental has maintain similars tendencies at the national until 1960. From this moment has been begun divergents tendencies. The region show the first signs of the depopulate process happened in others Spain's regions. In this paper show some characteristics of the population and his recent evolution.

Key words: Mancha Occidental. Population evolution. Demography structure. Depopulation.

RÉSUMÉ: Evolution récente de la population de la Mancha Occidental. La Mancha a presenté certaines tendances demographiques semblables a la nationale jusque 1960. Depuis lors, diverses tendances ont commencé. La région présente les premiers symptômes d'un procédé de dépopulation souffert dans d'autres regions espagnoles. Au cours de cet article, nous examinerons quelques principales caracteristiques de la population et sa récente evolution.

MoTS CLÉ: Mancha Occidental (Espagne Central). Evolution de la population. Structures demographiques. Depopulation. 
ANEXo I

CENSO DE POBLACIÓN DE 1981

\begin{tabular}{|c|c|c|c|c|c|c|c|c|c|c|c|c|c|c|}
\hline & POB & D & NV & $\mathbf{F}$ & CV & IJ & IV & TD & TAV & TAM & AGR & IND & $\mathrm{CON}$ & SER \\
\hline berca & 7 & 20 & 23 & 18 & 5 & 254,5 & 39,3 & 55 & 79,3 & 10,5 & 9,8 & 12,5 & 16,9 & 20,8 \\
\hline Alcáz & 25.541 & 38,3 & 410 & 263 & 147 & 199,6 & 50,1 & 62,6 & 73 & 14,6 & 13,8 & 19,2 & 10,1 & 56,1 \\
\hline bra & 1.474 & 2,5 & 8 & 15 & -7 & 110,1 & 90,9 & 73,8 & 66,6 & 5,5 & 51,5 & 4,9 & 15,5 & 28,1 \\
\hline Alm & 30 & 33,5 & 115 & 72 & 43 & 215,2 & 46,5 & 69,6 & 73,4 & 14,6 & 1 & 27,1 & 11,3 & 39,6 \\
\hline ren & 1.113 & 17,7 & 18 & 15 & 3 & 228,7 & 43,7 & 56,3 & 67,3 & 3,7 & 72,8 & 1,8 & 1,8 & 23,6 \\
\hline galle & 7.002 & 17,7 & 119 & 57 & 62 & 300,3 & 33,3 & 61,9 & 75,7 & 7,2 & 53,6 & 8,3 & 12,4 & 25,7 \\
\hline J10 & 1 & 113,1 & 150 & 87 & 63 & 290,1 & 34,5 & 63,2 & 74,3 & 12,6 & 50,5 & 14,3 & 7,7 & 27,1 \\
\hline am & 13.290 & 39,8 & 200 & 146 & 54 & 175 & 57,2 & 65,1 & 73,8 & 12 & 38,5 & 18,8 & 12 & 30,2 \\
\hline va & 2.425 & 25,3 & 38 & 30 & 8 & 255,4 & 39,2 & 73,5 & 73,6 & 6 & 32,9 & 12,2 & 20,7 & 34,2 \\
\hline asa & 1.545 & 49,8 & 21 & 17 & 4 & 173,4 & 57,7 & 49,9 & 74,9 & 6,9 & 6,7 & 10,2 & 16,7 & 16,4 \\
\hline asa & 1.018 & 9,2 & 9 & 11 & -2 & 108,8 & 91,9 & 86,4 & 61,3 & 1,2 & 84,9 & 6,2 & 2,1 & 6,8 \\
\hline asa & 650 & 9,6 & 11 & 5 & 6 & 186,7 & 53,6 & 65,8 & 75,1 & 3,9 & & 2,9 & 2,7 & 11,5 \\
\hline Dai & 16.249 & 37,1 & 321 & 150 & 81 & 229,2 & 43,6 & 66,9 & 71,4 & 16,1 & 4,6 & 19,8 & 17,5 & 37,2 \\
\hline Fer & & 11 & 16 & 19 & -3 & 128,9 & 77,6 & 76,9 & 70,3 & 2,6 & 5,1 & 3,9 & 13,7 & 37,3 \\
\hline ue & 5 & 3 & 59 & 46 & 13 & 168,1 & 59,5 & 64,5 & 74,5 & 11,2 & 7,7 & 7,7 & 9,4 & 25,2 \\
\hline $\mathrm{Her}$ & 77 & 31, & 107 & 75 & 32 & 230,2 & 43,4 & 71,3 & 72 & 12,9 & 9,7 & 15,3 & 16,3 & 28,7 \\
\hline$a b c$ & 6 & 20 , & 10 & 12 & -2 & 14 & 71,3 & 81,2 & 71,7 & 2,2 & 3,3 & & 3,3 & 3,3 \\
\hline Mes & 300 & 29 & 44 & 28 & 16 & 243,6 & 41 & 52 & 80,2 & 4,6 & & 10,7 & 8,9 & 20,4 \\
\hline Mal & 5 & 21 , & 128 & 100 & 28 & 166,2 & 60,2 & 62,9 & 76,9 & 5,2 & 9,9 & 7,1 & 6,4 & 16,6 \\
\hline a) & 7 & 35 , & 251 & 146 & 105 & 233,2 & 42,9 & 64 & 71,9 & 14,5 & 5,8 & 9,4 & 9,9 & 34,9 \\
\hline $\mathrm{Me}$ & & 45 & 110 & 51 & 59 & 298,9 & 33,5 & 60,8 & 74,6 & 16,4 & & 23,1 & 7,6 & 1,9 \\
\hline in & 22 & 31,7 & 20 & 33 & -13 & 188,7 & 53 & 64,6 & 72 & 6,2 & 6,6 & 9,2 & 10,3 & 23,9 \\
\hline Mot & & 31 & 82 & 37 & 45 & 272,5 & 36,7 & 63,2 & 74,7 & 14,4 & & 13,3 & 14,6 & 32,1 \\
\hline Mune & & 1 & 54 & 37 & 17 & 286,4 & 4,9 & 1,5 & 74,7 & 6,8 & 3,3 & 7,3 & 13,5 &, 9 \\
\hline $\mathrm{H}$ & & 2 & 23 & 18 & 5 & 142,5 & 70,2 & 56,8 & 71,4 & 9,7 & 3,2 & 1,6 & 15,7 & 39,4 \\
\hline edr & & 68, & 92 & 82 & 10 & 242,5 & 41,2 & 58 & 77,5 & 7,9 & & 22,8 & 14,2 & 34,1 \\
\hline Pedr & 59 & 28 & 93 & 48 & 45 & 285,5 & 35 & 65,7 & 76 & 3 & 5,1 & 4,4 & 6,9 & 24,5 \\
\hline 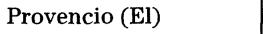 & & & 37 & 33 & 4 & 120,4 & 83,1 & 61,6 & 69,3 & 2,8 & 3,4 & 8,8 & 8,6 & 29,2 \\
\hline uer & & 23,1 & 1 & 17 & 0 & 102,2 & 97,8 & 56,5 & 68,7 & 13,9 & 37,3 & 11 & 14,9 & 36,8 \\
\hline an & 90 & 22,2 & 2 & 12 & 10 & 126 & 79,4 & 53,9 & 72,2 & 1,8 & 78 & 1,9 & 5,6 & 14,4 \\
\hline 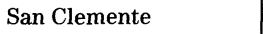 & & 23,9 & 91 & 54 & 37 & 262,9 & 38 & 62,6 & 76,1 & 16,8 & 32,8 & 13,1 & 16,4 & 36,7 \\
\hline an & & 28,9 & 9 & 2 & 7 & 155 & 64,5 & 83,7 & 75,4 & 3,3 & 72,3 & 2,1 & 12,8 & 12,8 \\
\hline Socu & 92 & 30,7 & 170 & 101 & 69 & 262 & 38,2 & 60,5 & 76,6 & 11,4 & 38,4 & 18,7 & 12,8 & 29,8 \\
\hline Solar & 13.568 & 101,3 & 179 & 111 & 68 & 292,5 & 34,2 & 63,4 & 76,1 & 14,8 & 37,8 & 26,5 & 8,4 & 26,9 \\
\hline 01 & 26.134 & 146 & 409 & 241 & 168 & 21 & 45,9 & 63,5 & 73 & 12,3 & 32,7 & 17,2 & 15,2 & 34,4 \\
\hline Torr & & 31,3 & 53 & 43 & 10 & 193,4 & 51,7 & 64 & 70,2 & 15,1 & 45,2 & 7,7 & 7 & 40,2 \\
\hline Vald & 25.059 & 51,4 & 422 & 222 & 200 & 206,4 & 48,5 & 67,7 & 73,5 & 15,6 & 17,4 & 21,9 & 8,7 & 51,4 \\
\hline ma & 20.172 & 23,4 & 342 & 149 & 193 & 297,8 & 33,6 & 64 & 80 & 13,7 & 27,2 & 19,1 & 15,9 & 37,5 \\
\hline illa & 9.008 & 31,9 & 138 & 79 & 59 & 210,5 & 47,5 & 64,3 & 69,8 & 5,1 & 37 & 10,7 & 30,4 & 21,6 \\
\hline Tilla & 2.878 & 43,6 & 55 & 26 & 29 & 241,1 & 41,5 & 67,8 & 80 & 8,4 & 47,9 & 12,2 & 8,6 & 30,3 \\
\hline lancha Occidental & 89.706 & 35,83 & 4.476 & 2.708 & 1.768 & 211,1 & 52,1 & 64,9 & 73,5 & 9,2 & 47,7 & 11,6 & 11,6 & 28,8 \\
\hline
\end{tabular}

Fuentes: Censos de Población. Elaboración propia.

NOTA: POB: población; D: Densidad (hab./km²); NV: Nacidos vivos; F: Fallecidos; CV: Crecimientoo vegetativo; IJ: Índice de Juventud (\%); IV: Índice de Vejez (\%); TD: Tasa de Dependencia (\%); TAV: Tasa de Actividad Varones (\%); TAM: Tasa de Actividad Mujeres (\%); AGR: Población ocupada en Agricultura (\%); IND: Población ocupada en Industria (\%); CON: Población ocupada en Construcción (\%); SERV: Población ocupada en Servicios (\%). 


\section{ANEXo II}

CENSO DE POBLACIÓN DE 1991

\begin{tabular}{|c|c|c|c|c|c|c|c|c|c|c|c|c|c|c|}
\hline & POB & $\mathbf{D}$ & NV & $\mathbf{F}$ & $\mathbf{C V}$ & $\mathbf{I J}$ & IV & TD & TAV & TAM & AGR & IND & CON & SER \\
\hline Alberca de Záncara (La) & 1.875 & 18,6 & 16 & 12 & 4 & 117 & 85,5 & 60 & 68 & 13,6 & 48,9 & 14,1 & 15,5 & 21,5 \\
\hline Alcázar de San Juan & 25.996 & 39 & 273 & 265 & 8 & 146,1 & 68,4 & 60,6 & 67,3 & 25,5 & 5,6 & 23,1 & 10,2 & 61,1 \\
\hline Alhambra & 1.423 & 2,5 & 15 & 11 & 4 & 137 & 73 & 57,4 & 73,3 & 17,2 & 31,3 & 18,8 & 34,7 & 15,2 \\
\hline Almagro & 8.356 & 33,4 & 132 & 78 & 54 & 191,7 & 52,2 & 62,5 & 72,9 & 22,6 & 5,9 & 30,2 & 12,2 & 51,6 \\
\hline Arenas de San Juan & 1.065 & 16,9 & 16 & 7 & 9 & 131,9 & 75,8 & 67,5 & 62,7 & 6,9 & 23,3 & 7,6 & 36,9 & 32,2 \\
\hline Argamasilla de Alba & 6.495 & 16,4 & 95 & 46 & 49 & 188,4 & 53,1 & 57,5 & 71,3 & 20,7 & 30,4 & 14,7 & 19,8 & 35,1 \\
\hline Bolaños de Calatrava & 10.271 & 116,7 & 120 & 101 & 19 & 206,1 & 48,5 & 58 & 73,9 & 20,4 & 28,4 & 19 & 15 & 37,6 \\
\hline Campo de Criptana & 13.727 & 41,1 & 171 & 151 & 20 & 136,4 & 73,3 & 62 & 69,8 & 20,9 & 20,2 & 22,1 & 23,2 & 34,5 \\
\hline Carrión de Calatrava & 2.448 & 25,5 & 28 & 26 & 2 & 152,6 & 65,5 & 62 & 67,2 & 23,5 & 14,8 & 10,4 & 27,1 & 47,7 \\
\hline Casas de Fernando A. & 1.407 & 45,4 & 19 & 9 & 10 & 94,8 & 105 & 52,9 & 65,8 & 13,2 & 45,6 & 10 & 14,2 & 30,2 \\
\hline Casas de Haro & 898 & 8,1 & 12 & 12 & 0 & 75,3 & 132 & 63 & 62,4 & 18 & 49,8 & 14,5 & 11,8 & 23,9 \\
\hline Casas de los Pinos & 584 & 8,6 & 3 & 5 & -2 & 76,1 & 131 & 51,7 & 65,8 & 11,4 & 56,8 & 4 & 11,9 & 27,3 \\
\hline Daimiel & 16.668 & 38,1 & 232 & 165 & 67 & 145,2 & 68,9 & 59,6 & 69 & 24,8 & 10,8 & 19,4 & 29,9 & 39,8 \\
\hline Fernáncaballero & 1.040 & 10 & 10 & 11 & -1 & 721 & 138 & 70,5 & 62,7 & 9,6 & 34,5 & 18,3 & 22,6 & 24,6 \\
\hline Fuente el Fresno & 3.546 & 29,6 & 48 & 42 & 6 & 134,1 & 74,6 & 63,1 & 67,3 & 17 & 34,3 & 11 & 23,5 & 31,2 \\
\hline Herencia & 6.894 & 30,4 & 97 & 75 & 22 & 136,3 & 73,4 & 61,8 & 73,2 & 16,2 & 24,2 & 19,1 & 28,8 & 27,9 \\
\hline Labores (Las) & 695 & 20,4 & 2 & 5 & -3 & 84,7 & 118 & 66,3 & 58,4 & 11,6 & 27 & 8,1 & 33,8 & 31,1 \\
\hline Mesas (Las) & 2.627 & 30,2 & 26 & 26 & 0 & 139 & 71,9 & 59,5 & 69,9 & 15,3 & 35,1 & 14,3 & 15,9 & 34,7 \\
\hline Malagón & 7.888 & 21,7 & 94 & 101 & -7 & 102 & 98 & 69,6 & 70,2 & 24,4 & 49,1 & 9,8 & 15,8 & 25,3 \\
\hline Manzanares & 17.916 & 36,2 & 225 & 181 & 44 & 151,7 & 65,9 & 62,8 & 62,6 & 22,1 & 21,7 & 17,3 & 18,7 & 42,3 \\
\hline Membrilla & 6.706 & 46,6 & 80 & 51 & 29 & 159,1 & 62,8 & 65,6 & 72,3 & 21 & 8,1 & 21,9 & 11,1 & 59 \\
\hline Minaya & 1.940 & 27,7 & 12 & 23 & -11 & 89,2 & 112 & 63,7 & 63,5 & 8,4 & 35,3 & 9,3 & 19,5 & 36 \\
\hline Mota del Cuervo & 5.602 & 31,8 & 63 & 46 & 17 & 179,6 & 55,7 & 61,1 & 70,5 & 18,2 & 21,9 & 12,8 & 24,8 & 40,4 \\
\hline Munera & 4.087 & 17,8 & 52 & 46 & 6 & 123,7 & 80,8 & 58,5 & 68,9 & 21 & 36 & 15,5 & 21,3 & 27,1 \\
\hline El Pedernoso & 1.412 & 25,2 & 10 & 15 & -5 & 85,3 & 117 & 62,1 & 64,3 & 14,4 & 34,5 & 15 & 15,3 & 35,3 \\
\hline Pedro Muñoz & 7.080 & 70,1 & 90 & 67 & 23 & 158,1 & 63,2 & 55,9 & 70,5 & 13,3 & 19,6 & 28,8 & 14,7 & 36,9 \\
\hline Pedroñeras (Las) & 6.545 & 29,1 & 89 & 50 & 39 & 178 & 56,2 & 60,9 & 71,9 & 16,9 & 50,8 & 9,4 & 7,8 & 32 \\
\hline Provencio (El) & 2.720 & 26,9 & 14 & 30 & -16 & $79,\left.4\right|_{1}$ & 125 & 65,1 & 58,6 & 12,5 & 35,1 & 16,5 & 14,6 & 33,9 \\
\hline Puerto Lápice & 1.013 & 18,4 & 11 & 9 & 2 & 103,2 & 96,9 & 59 & 72,5 & 17,4 & 22,4 & 19 & 19 & 39,6 \\
\hline San Carlos del Valle & 1.298 & 22,4 & 12 & 16 & -4 & 109,5 & 91,3 & 68,6 & 63,2 & 6,2 & 60 & 4,8 & 13,3 & 21,8 \\
\hline San Clemente & 6.258 & 22,5 & 64 & 52 & 12 & 155,1 & 64,5 & 58,3 & 70,7 & 20,7 & 23,6 & 13,8 & 19,5 & 43,1 \\
\hline Santa María de los & 903 & 21 & 6 & 15 & -9 & 71,9 & 139 & 57,6 & 63,4 & 7,5 & 48,8 & 9,3 & 23,6 & 18,3 \\
\hline Socuéllamos & 11.388 & 30,4 & 146 & 125 & 21 & 141,1 & 70,9 & 58,1 & 69,7 & 20,2 & 21,3 & 22,7 & 20,9 & 35,1 \\
\hline Solana (La) & 14.160 & 105,7 & 221 & 132 & 89 & 169,8 & 58,9 & 62,7 & 70,9 & 20,4 & 23,1 & 23,4 & 26,9 & 26,7 \\
\hline Tomelloso & 28.632 & 160 & 370 & 263 & 107 & 161,5 & 61,9 & 61,6 & 72,1 & 20,7 & 17,2 & 24,2 & 22,5 & 36 \\
\hline Torralba de Calatrava & 3.042 & 29,8 & 29 & 35 & -6 & 98,5 & 101 & 62,9 & 62 & 17,7 & 26 & 12,5 & 19,2 & 42,4 \\
\hline Valdepeñas & 25.530 & 52,3 & 358 & 255 & 103 & 151 & 88,2 & 80 & 88,8 & 24,4 & 7,8 & 25,8 & 13 & 53,8 \\
\hline Villarrobledo & 20.705 & 24 & 272 & 181 & 91 & 185,8 & 53,8 & 56,7 & 72,1 & 21,4 & 14,6 & 23,7 & 21,3 & 40,4 \\
\hline Villarrubia de los Ojos & 9.461 & 33,5 & 128 & 83 & 45 & 142,7 & 70,1 & 59,9 & 64,1 & 13 & 13,9 & 12,8 & 45,3 & 28 \\
\hline Villarta de San Juan & 2.969 & 45 & 31 & 27 & 4 & 150,4 & 66,5 & 55,9 & 74 & 14,3 & 36,5 & 18,9 & 18,2 & 26,3 \\
\hline Mancha Occidental & 293.270 & 35,73 & 3.692 & 2.850 & 842 & 132,8 & 82,3 & 61,1 & 67,9 & 17,1 & 58,9 & 16,1 & 20,3 & 34,7 \\
\hline
\end{tabular}

Fuentes: Censos de Población. Elaboración propia.

NOTA: POB: población; D: Densidad (hab./km²); NV: Nacidos vivos; F: Fallecidos; CV: Crecimientoo vegetativo; IJ: Índice de Juventud (\%); IV: Índice de Vejez (\%); TD: Tasa de Dependencia (\%); TAV: Tasa de Actividad Varones (\%); TAM: Tasa de Actividad Mujeres (\%); AGR: Población ocupada en Agricultura (\%); IND: Población ocupada en Industria (\%); CON: Población ocupada en Construcción (\%); SERV: Población ocupada en Servicios (\%). 